\title{
La melatonina reduce la respuesta de cortisol al ACTH en humanos
}

\author{
Carmen Campino ${ }^{1 a}$, Francisco Valenzuela ${ }^{2 b}$, Eugenio Arteaga ${ }^{1}$, \\ Claudia Torres-Farfán ${ }^{2 c}$, Cristián Trucco ${ }^{3}$, Alfredo Velasco ${ }^{3}$, \\ Sergio G uzmán ${ }^{3}$, María Serón-Ferré2,4d. \\ Melatonin reduces cortisol response \\ to $A C T H$ in humans
}

\begin{abstract}
Background: Melatonin receptors are widely distributed in human tissues but they have not been reported in human adrenal gland. Aim: To assess if the human adrenal gland expresses melatonin receptors and if melatonin affects cortisol response to ACTH in dexamethasone suppressed volunteers. Material and methods: Adrenal glands were obtained from 4 patients undergoing unilateral nephrectomy-adrenalectomy for renal cancer. Expression of mRNA MT1 and MT2 melatonin receptors was measured by Reverse Transcriptase Polymerase Chain Reaction (RT-PCR). The effect of melatonin on the response to intravenous (i.v.) ACTH was tested (randomized cross-over, double-blind, placebo-controlled trial) in eight young healthy males pretreated with dexamethasone (1 mg) at 23:00 h. On the next day, at 08:00 h, an i.v. line was inserted, at 08:30 h, and after a blood sample, subjects ingested $6 \mathrm{mg}$ melatonin or placebo. At 09:00 h, 1-24 ACTH (Cortrosyn, $1 \mu \mathrm{g} / 1.73 \mathrm{~m}^{2}$ body surface area) was injected, drawing samples at $0,15,30,45$ and 60 minutes after. Melatonin, cortisol, cortisone, progesterone, aldosterone, DHEA-S, testosterone and prolactin were measured by immunoassay. Results: The four adrenal glands expressed only MT1 receptor mRNA. Melatonin ingestion reduced the cortisol response to ACTH from $14.6 \pm 1.45 \mu \mathrm{g} / \mathrm{dl}$ at 60 min in the placebo group to $10.8 \pm 1.2 \mu \mathrm{g} / \mathrm{dl}$ in the melatonin group ( $\mathrm{p}<0.01$ mixed model test). It did not affect other steroid hormone levels and abolished the morning physiological decline of prolactin. Conclusions: The expression of MT1 melatonin receptor in the human adrenal, and the melatonin reduction of ACTH-stimulated cortisol production suggest a direct melatonin action on the adrenal gland (Rev Méd Chile 2008; 136: 1390-7).
\end{abstract}

(Key words: Adrenocorticotropic hormone; Hydrocortisone; Melatonin)
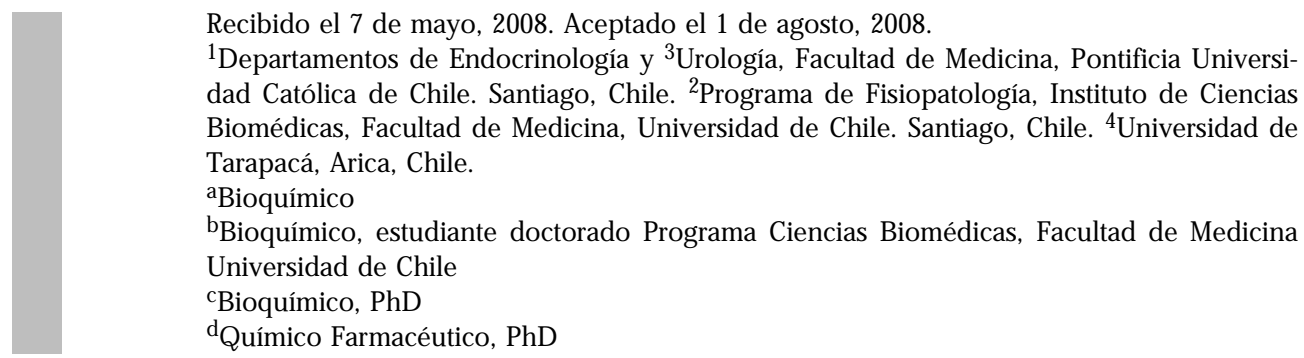

Correspondencia a: Carmen Campino. Departamento de Endocrinología, Escuela de Medicina, Pontificia Universidad Católica de Chile. Lira 85, 5piso, Santiago, Chile. Fax: 56-2-6385675. E mail: ccampino@med.puc.cl 
L a neurohormona melatonina es un transductor meuroendocrino del ciclo luz-oscuridad. Su secreción aumenta con el inicio de la oscuridad, alcanzando su máximo en la mitad de la noche y cayendo abruptamente al amanecer, indicando tanto el comienzo y la duración de la noche como las estaciones del año ${ }^{1}$. Así el ritmo diario de melatonina es una señal cronobiótica, es decir, permite al individuo ajustar sus funciones fisiológicas al ciclo luz-oscuridad imperante ${ }^{2}$.

La identificación de receptores de melatonina en una variedad de tejidos en humanos y en animales ha estimulado la búsqueda de otras funciones fisiológicas de la melatonina ${ }^{3}$. Recientemente ha despertado interés su posible acción sobre la esteroidogénesis. La melatonina actúa a través de 2 receptores de membrana llamados MT1 y MT2. En humanos, ambos receptores de melatonina están presentes en tejidos esteroidogénicos como la placenta y células de la granulo$\mathrm{sa}^{4,5}$. Adicionalmente, estudios realizados en animales demuestran la presencia solo del receptor MT1 en las células de Leydig de la rata y hamster y en la glándula suprarrenal de rata adulta y de fetos y adultos de primates no humanos ${ }^{6-10}$. En esta última glándula, la melatonina inhibe la expresión de genes circadianos ${ }^{11}$ y también la producción de cortisol estimulada por ACTH ${ }^{9,10}$.

En humanos, los ritmos circadianos de melatonina y de cortisol en plasma tienen fases opuestas, es decir el alza nocturna de melatonina coincide con los valores bajos del ritmo de cortisol y viceversa ${ }^{12}$. Por otra parte, la supresión aguda de melatonina producida por una breve exposición a luz brillante al final de la noche se asocia con un alza brusca del cortisol plasmático y salival ${ }^{13,14}$. Sin embargo, estudios realizados en mujeres y hombres, tratados con distintas dosis de melatonina, a distintas horas del día, en una o varias ocasiones, o estudiando el efecto de la administración de melatonina sobre la producción de cortisol estimulada por ACTH, tanto exógena como endógena, no han mostrado efectos claros sobre la concentración plasmática de cortisol $^{15-19}$. Dada la compleja regulación de la corteza suprarrenal, es posible que en estos estudios un potencial efecto inhibidor de melatonina sobre la producción de cortisol haya sido enmascarado por los mecanismos de feedback entre cortisol y ACTH ${ }^{20}$.
El presente estudio fue diseñado para investigar la hipótesis de que la melatonina modula la respuesta de cortisol a ACTH en humanos. Para ello investigamos: 1) Si la glándula suprarrenal humana expresa receptores de melatonina; y 2) $\mathrm{Si}$ una dosis única de melatonina [6 $\mathrm{mg}$, dentro de los márgenes usados como agente cronobiótico ${ }^{2}$ ], tiene un efecto inhibitorio agudo sobre la producción de cortisol estimulada por ACTH en sujetos suprimidos con dexametasona para evitar la interferencia del eje hipotálamo-hipófisis.

\section{SujETOS Y MÉTODOS}

Los protocolos, aprobados por el Comité de Ética de la Facultad de Medicina de la Pontificia Universidad Católica de Chile fueron explicados a los participantes, quienes entregaron su consentimiento informado de acuerdo a las pautas de la Declaración de Helsinki.

Identificación de los receptores de melatonina en glándula suprarrenal humana por RT-PCR. Se obtuvo tejido suprarrenal de 4 pacientes sometidos a nefrectomía-suprarrenalectomía por cáncer renal. Aproximadamente $100 \mathrm{mg}$ de corteza suprarrenal fueron guardados en TRIzol (Life Technology Inc., Rockville, MD, USA) y el resto fue enviado a Anatomía Patológica. Tres suprarrenales correspondían a tejido normal y la cuarta a un adenoma. En un caso se removió tejido renal normal adyacente al tumor que fue usado como control positivo $^{21}$. El ARN total fue extraído usando TRIzol y la expresión de mARN de los receptores MT1 y MT2 de melatonina y 18S-rARN en glándula suprarrenal y en tejido renal fue analizada por RTPCR usando los partidores y la metodología previamente descrita ${ }^{9,10}$. La banda correspondiente al producto de PCR del receptor MT1 fue cortada, reamplificada, purificada y luego secuenciada en el Departamento de Ecología, Facultad de Ciencias Biológicas, Pontificia Universidad Católica de Chile, determinándose la identidad con la secuencia reportada para el receptor MT1 humano usando el programa BLASTN 2.2.1 (www.ncbi.nlm.nih.gov).

Efecto agudo de la melatonina sobre la esteroidogénesis suprarrenal en respuesta a ACTH. Se reclu- 
taron 8 hombres clínicamente sanos, no obesos (Índice de masa comoral, $\bar{x} \pm E E=24,8 \pm 0,75 \mathrm{~kg} / \mathrm{m}^{2}$ ), sin trastornos de sueño, no fumadores, sin ingesta de medicamentos, de edades entre 24-34 años $(\overline{\mathrm{x}} \pm \mathrm{EE}=27,25 \pm 1,23$ años) y con función suprarrenal normal evaluada en 1 muestra de sangre tomada a las 09:00 horas, en ayunas, dos semanas previas al estudio (muestras preestudio). La concentración promedio de ACTH fue 14,3 \pm $1,7 \mathrm{pg} / \mathrm{ml}$ (valor de referencia, VR: $10-60 \mathrm{pg} / \mathrm{ml}$ ); cortisol 11,1 $\pm 1,4 \mu \mathrm{g} / \mathrm{dl}$ (VR: 6,4-15 $\mu \mathrm{g} / \mathrm{dl}$ ); DHEAS 403,1 \pm 53,4 $\mu \mathrm{g} / \mathrm{dl}$ (VR: 281-606 $\mu \mathrm{g} / \mathrm{dl}$ ) y progesterona $68,4 \pm 4,35 \mathrm{ng} / \mathrm{dl}$ (VR: 10,1-120 ng/ dl). El día previo al inicio del estudio, los sujetos ingirieron $1 \mathrm{mg}$ de dexametasona (2 tabletas de 0,5 mg, Oradexon, Organon, Holland) a las $23 \mathrm{~h}$. $\mathrm{Al}$ día siguiente, los sujetos ingresaron a las 07:45 $h$, en ayunas, a la sala metabólica del Hospital Clínico de la Pontificia Universidad Católica de Chile, permaneciendo en posición supina durante todo el protocolo. La iluminación de la sala fue de 300 lux de luz artificial al nivel de los ojos de los voluntarios. A las 08:00 h se les instaló una bránula heparinizada en una vena antecubital de un antebrazo y a las 08:30 $\mathrm{h}$ se les extrajo una muestra de sangre (tiempo: $30 \mathrm{~min}$ ). Inmediatamente después los sujetos ingirieron 2 cápsulas de $3 \mathrm{mg}$ de melatonina pura (Sigma, St. Louis, USA) o placebo (almidón), preparadas en la Farmacia Cruz Verde, Santiago, Chile. Treinta minutos después, previa extracción de otra muestra de sangre (tiempo 0), se les inyectó en el otro antebrazo una microdosis, recién preparada, de 1-24 ACTH [1 $\mu \mathrm{g} / 1,73 \mathrm{~m}^{2}$ de superficie corporal; Cortrosyn, Organon, Holanda ${ }^{19,20}$, tomándose muestras de sangre 15, 30, 45 y 60 min después de la inyección. Los sueros o plasmas se separaron inmediatamente, guardándose en alícuotas a $-20^{\circ} \mathrm{C}$ hasta su análisis. En todas las muestras se midió melatonina, cortisol, cortisona, progesterona, DHEA-S y prolactina. La medición de prolactina tuvo por objeto servir de control del estudio dado que se conoce que melatonina induce aumento de sus niveles circulantes ${ }^{15,17,18}$. Además se midió aldosterona y testosterona en las muestras de sangre extraídas a -30 min y 60 minutos post ACTH. Los protocolos se realizaron separados por 7 días y el orden "droga activa" versus placebo se decidió al azar en protocolo cruzado, doble ciego. Efectuamos la prueba de ACTH a las
08:30 h, ya que la respuesta de la glándula suprarrenal a ACTH es mayor a esta hora $22-24$.

ACTH se midió por inmunoensayo automatizado, en un equipo Immulite 2000 de DPC (Diagnostic Product Corporation, Morganville, New Jersey, USA). Melatonina, cortisol, cortisona y testosterona se

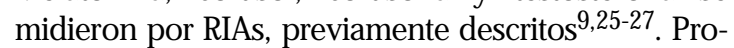
gesterona, aldosterona (RIA), prolactina (IRMA) y DHEA-S (RIA) se midieron usando kits comenciales DPC (Diagnostic Product Corporation, Los Angeles, CA, USA) y por DSL (Diagnostic Systems Laboratories, Inc. Texas, USA). Los coeficientes de variación intra e interensayo fueron inferiores a $15 \%$.

Análisis de los resultados. Las concentraciones hormonales se expresaron como promedio \pm E.E. El curso temporal de la concentración de melatonina y prolactina en plasma y la respuesta a ACTH de los distintos esteroides fue analizada por ANOVA de una vía para muestras repetidas seguida de test de Tukey usando el programa GraphPad Prism software (version 4.0; GraphPad Software Inc., San Diego, CA, USA). Las comparaciones entre los grupos que tomaron placebo y melatonina se realizaron por el análisis de modelos mixtos ${ }^{28}$ del programa Statistical Analysis System (SAS; Pre-Production version 9.00 level TS 00MO, NC; USA). Las diferencias se consideraron significativas cuando $\mathrm{p}<0,05$.

\section{Resultados}

Identificación del receptor de melatonina en la glándula suprarrenal humana por RT-PCR. La glándula suprarrenal humana expresa el receptor MT1 de melatonina como se observa en la Figura 1. Productos de PCR del tamaño correspondiente al receptor MT1 humano se obtuvieron tanto en las 3 glándulas suprarrenales normales como en el adenoma suprarrenal. La secuencia de este producto fue idéntica a la secuencia entre las bases 477-912 del mARN del receptor MT1 de melatonina correspondiente a los dominios transmembrana 3-6 de la proteína (Genbank GI: 60498974 for homo sapiens melatonin receptor 1 A (MTNR 1 A) $\mathrm{mARN}^{29}$. En contraste, no se detectó la expresión del mARN del receptor MT2 en suprarrenal, mientras que expresión de MT1 y MT2 se detectó en el tejido renal usado como control positivo ${ }^{21}$. 


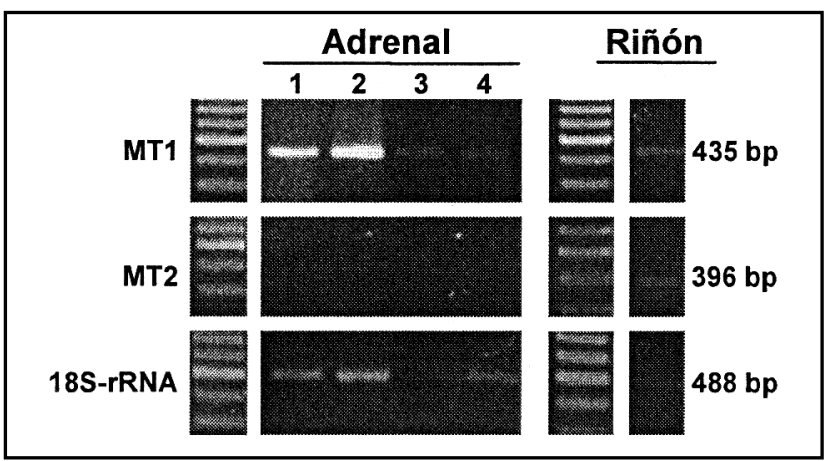

Figura 1. Expresión del mARN de los receptores MT1 y MT2 de melatonina y de 18S-rARN en glándula supramenal humana y en tejido renal analizado por RT-PCR. Lúnea 1: Adenoma supramenal. Camiles: 2, 3, 4: Glándulas suprarrenales normales. En las muestras de tejido supramenal, los productos de PCR para los receptores MT1 y MT2 de melatonina se obtuvieron a partir de concentraciones de cADN equivalentes a $300 \mathrm{ng}$ de ARN y para 18S-rARN a $150 \mathrm{ng}$ de ARN. En el tejido renal, los productos de PCR para los receptores MT1 y MT2 de melatonina y para 18SrARN se obtuvieron a partir de concentraciones de cADN equivalentes a $150 \mathrm{ng}$ de ARN.

\section{Tabla 1. C urso temporal de la concentración de melatonina en plasma $(\mathrm{pg} / \mathrm{ml})$ en sujetos suprimidos con} dexametasona $(n=8)$ después de ingerir una dosis única de melatonina $(6 \mathrm{mg})$ o placebo

\begin{tabular}{|c|c|c|c|}
\hline Tiempo & (min) & Placebo & Melatonina \\
\hline Basal & $\begin{array}{r}(-30) \\
(0) \\
(+15) \\
(+30) \\
(+45) \\
(+60)\end{array}$ & $\begin{array}{l}40,0 \pm 7,1 \\
45,9 \pm 2,2 \\
47,1 \pm 5,9 \\
52,1 \pm 3,7 \\
50,8 \pm 6,8 \\
47,7 \pm 7,1\end{array}$ & $\begin{array}{rcc}45,5 & \pm & 5,4 \\
7746,9 & \pm 1707,4^{*, \dagger} \\
9316,8 & \pm 1836,8^{*,+} \\
10778,1 & \pm 2210,1 \\
8458,7 & \pm 1341,1^{*,} \\
6978,1 & \pm 1279,1^{*,+}\end{array}$ \\
\hline
\end{tabular}

Los valores corresponden a promedio \pm EE. El tiempo indicado entre paréntesis se refiere al tiempo de la administración de ACTH (ver métodos). La melatonina fue ingerida inmediatamente después de extraer la muestra basal.

*Distinto del basal $(\mathrm{p}<0,05$ ANOVA de una vía para muestras repetidas y prueba de Tukey para encontrar las diferencias.

†Distinto del placebo a los mismos intervalos de tiempo ( $\mathrm{p}<0,01$ análisis de modelos mixtos).

Efecto agudo de la melatonina sobre la esteroidogénesis suprarrenal en respuesta a ACTH. La ingesta de melatonina en la dosis utilizada para lograr efectos cronobióticos ${ }^{2,30}$, provocó un alza en las concentraciones plasmáticas de melatonina las que se mantuvieron a lo largo del test de ACTH (Tabla 1). Como era predecible, el tratamiento con dexametasona redujo las concentraciones de cortisol, aldosterona, progesterona, DHEA-S y cortisona.
La melatonina disminuyó la respuesta del cortisol al ACTH obteniéndose valores más bajos que en el grupo placebo a los 45 y 60 min (Figura 2; p $<0,05$, análisis de modelos mixtos). La melatonina no modificó las concentraciones basales de ninguno de los esteroides ni la respuesta de cortisona, aldosterona y progesterona al ACTH (Tabla 2). DHEA-S y testosterona no respondieron a la inyección de ACTH y la melatonina no modificó las concentraciones de estos 2 esteroides (Tabla 2). 


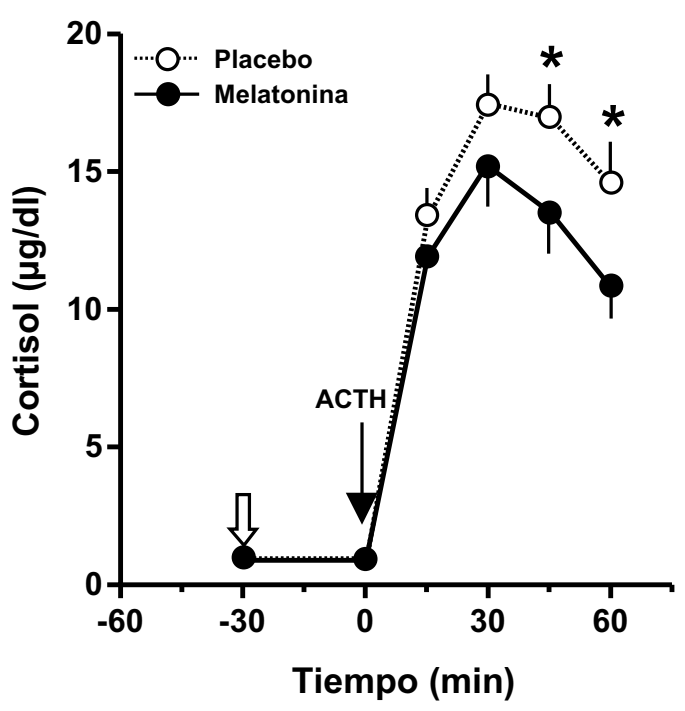

Figura 2. Efecto de melatonina sobre la concentración plasmática de cortisol $(\overline{\mathrm{x}} \pm \mathrm{EE}, \mathrm{n}=8)$ en respuesta a 1-24 ACTH $\left(1 \mu \mathrm{g} / 1,73 \mathrm{~m}^{2}\right.$ de superficie corporal, i.v.) en sujetos suprimidos con dexametasona. Placebo (círculos vacíos) o una dosis única de melatonina ( $6 \mathrm{mg}$, círculos llenos) fueron ingeridos al tiempo que indica la pequeña flecha blanca.

* Diferente a ACTH con melatonina ( $\mathrm{P}<0,01$, análisis de modelos mixtos).

Tabla 2. Efecto de la melatonina sobre el curso temporal de las concentraciones plasmáticas de cortisona (E), progesterona $\left(\mathrm{P}_{4}\right)$, aldosterona (Aldo), D H EA-S y testosterona (T) inducida por ACT H, en sujetos suprimidos con dexametasona $(n=8)$

\begin{tabular}{|c|c|c|c|c|c|c|c|c|c|c|}
\hline \multirow[b]{2}{*}{$\begin{array}{l}\text { Tiempo } \\
\text { minutos }\end{array}$} & \multicolumn{5}{|c|}{ Placebo } & \multicolumn{5}{|c|}{ Melatonina } \\
\hline & $\begin{array}{c}\mathbf{E} \\
\mu \mathrm{g} / \mathrm{dl}\end{array}$ & $\begin{array}{c}P_{4} \\
n g / d l\end{array}$ & $\begin{array}{l}\text { Aldo } \\
\mathrm{ng} / \mathrm{dl}\end{array}$ & $\begin{array}{c}\text { DHEA-S } \\
\mu \mathrm{g} / \mathrm{dl}\end{array}$ & $\begin{array}{c}\mathrm{T} \\
\mathrm{ng} / \mathrm{dl}\end{array}$ & $\underset{\mu g / d l}{E}$ & $\begin{array}{c}\mathrm{P}_{4} \\
\mathrm{ng} / \mathrm{dl}\end{array}$ & $\begin{array}{l}\text { Aldo } \\
\mathrm{ng} / \mathrm{dl}\end{array}$ & $\begin{array}{c}\text { DHEA-S } \\
\mu \mathrm{g} / \mathrm{dl}\end{array}$ & $\begin{array}{c}\mathrm{T} \\
\mathrm{ng} / \mathrm{dl}\end{array}$ \\
\hline-30 & $0,44 \pm 0,03$ & $52,0 \pm 5,2$ & $5,5 \pm 1,0$ & $228,3 \pm 21,2$ & $509,8 \pm 28,8$ & $0,38 \pm 0,04$ & $52,7 \pm 5,9$ & $3,7 \pm 1,0$ & $210,9 \pm 18,1$ & $510,6 \pm 29,9$ \\
\hline 0 & $0,38 \pm 0,04$ & $51,7 \pm 5,7$ & NM & $218,1 \pm 20,2$ & NM & $0,38 \pm 0,03$ & $51,8 \pm 6,5$ & NM & $202,8 \pm 18,6$ & NM \\
\hline+15 & $1,61 \pm 0,16^{*}$ & $100,7 \pm 9,5^{*}$ & NM & $239,0 \pm 21,0$ & NM & $1,45 \pm 0,10^{*}$ & $99,5 \pm 5,9 *$ & NM & $207,5 \pm 14,6$ & NM \\
\hline+30 & $2,22 \pm 0,10^{*}$ & $112,3 \pm 7,0^{*}$ & NM & $253,3 \pm 25,2$ & NM & $2,15 \pm 0,12 *$ & $101,5 \pm 9,5^{*}$ & NM & $215,1 \pm 16,0$ & NM \\
\hline+45 & $2,46 \pm 0,11^{*}$ & $100,6 \pm 5,6^{*}$ & NM & $240,6 \pm 18,8$ & NM & $2,29 \pm 0,15^{*}$ & $92,6 \pm 7,8^{*}$ & NM & $214,4 \pm 18,9$ & NM \\
\hline+60 & $2,49 \pm 0,08^{*}$ & $89,5 \pm 6,9 *$ & $11,8 \pm 1,6^{*}$ & $244,6 \pm 24,8$ & $480,9 \pm 21,2$ & $2,32 \pm 0,16^{*}$ & $79,8 \pm 4,5^{*}$ & $9,1 \pm 1,5^{*}$ & $218,8 \pm 25,8$ & $483,0 \pm 31,7$ \\
\hline
\end{tabular}

Valores corresponden a promedio \pm EE. Una dosis única de melatonina $(6 \mathrm{mg})$ o placebo fue ingerida $30 \mathrm{~min}$ antes de la inyección de ACTH. NM: no medido.

$*_{p}<0,05$ versus tiempo -300 cero.

Prolactina. La prolactina mostró un progresivo descenso a lo largo de los 60 min de duración del test de ACTH en el grupo placebo (Tabla 3). Esta disminución fue revertida por el tratamiento con melatonina, obteniéndose concentraciones más altas de prolactina que en el grupo placebo desde los 30 a los 60 min post ACTH. 
Tabla 3. Curso temporal de las concentraciones séricas de prolactina $(\mathrm{pg} / \mathrm{ml})$ en sujetos suprimidos con dexametasona ( $n=8)$, después de ingerir una dosis única de melatonina (6 mg) o placebo

\begin{tabular}{|rrll|}
\hline \multicolumn{2}{|c}{ Tiempo (min) } & Placebo & Melatonina \\
\hline Basal & $(-30)$ & $6,5 \pm 0,9$ & $6,5 \pm 0,9$ \\
& $(0)$ & $6,0 \pm 0,9$ & $6,5 \pm 0,9$ \\
& $(+15)$ & $5,8 \pm 0,9^{*}$ & $6,5 \pm 0,9$ \\
& $(+30)$ & $5,4 \pm 0,9^{*}$ & $6,9 \pm 0,7^{\dagger}$ \\
& $(+45)$ & $5,3 \pm 0,9^{*}$ & $6,7 \pm 0,8^{\dagger}$ \\
& $(+60)$ & $5,1 \pm 0,8^{*}$ & $6,5 \pm 0,3^{\dagger}$ \\
\hline
\end{tabular}

Valores corresponden a promedio \pm EE. El tiempo indicado entre paréntesis corresponde al tiempo al que se inyectó ACTH (ver métodos). Melatonina fue ingerida inmediatamente después de extraída la muestra basal.

*Diferente al basal ( $\mathrm{p}<0,05$ ANOVA de una vía para muestras repetidas, seguido de análisis de Tukey para encontrar las diferencias).

${ }^{\dagger}$ Distinto al placebo al mismo intervalo de tiempo ( $p<0,01$ análisis de modelos mixtos).

\section{DisCUSIÓN}

Nuestro estudio demuestra la presencia del receptor MT1 de melatonina en la glándula suprarenal humana y un efecto agudo de la melatonina disminuyendo la producción de cortisol estimulada por ACTH en voluntarios pretratados con dexametasona.

Hasta ahora no había sido investigada la presencia de receptores de melatonina en la glándula suprarrenal humana. En este estudio, encontramos por primera vez la expresión del mARN del receptor MT1 en las 3 muestras analizadas de tejido suprarrenal normal y en una de adenoma suprarrenal. La expresión del mARN del receptor MT2 no se detectó en ninguna de las muestras de tejido suprarrenal, usando las mismas concentraciones de cADN usadas para analizar el receptor MT1. En cambio detectamos la expresión de mARN para el receptor MT1 y MT2 en el tejido humano renal como ha sido previamente publicado ${ }^{21} \mathrm{y}$ fue usado como control positivo. Estos resultados son similares a los observados por nosotros en la corteza suprarrenal de la rata y del mono capuchino ${ }^{8-10}$.
En este estudio, las concentraciones de melatonina alcanzadas en el plasma fueron más altas que las descritas por Fischer ${ }^{30}$ dando una dosis similar, lo que podría deberse a diferencias en las condiciones experimentales, ya que nosotros administramos la melatonina a las 08:30 h después de un ayuno de $12 \mathrm{~h}$ y Fischer dio la melatonina (5 mg) a las 22:00 $\mathrm{h}$ sin especificar la hora de la última comida.

En las condiciones en que se realizó este estudio, el tratamiento con melatonina mostró un efecto agudo in vivo reduciendo la producción de cortisol en respuesta al ACTH. Este efecto fue ejercido sin afectar las concentraciones basales de cortisol, suprimidas por la administración de dexametasona la medianoche anterior. La disminución de la respuesta del cortisol fue detectada a los 45 y 60 min después de la inyección de ACTH, fue de magnitud variable y se observó en 7 de los 8 sujetos seleccionados. La melatonina no mostró efectos significativos en la respuesta a ACTH sobre los otros esteroides suprarenales medidos: progesterona y aldosterona como tampoco sobre cortisona. Las concentraciones plasmáticas de estos esteroides al igual que las de cortisol disminuyeron con el tratamiento con dexametasona comparado con los valores obtenidos en las muestras preestudio o con valores normales basales obtenidos en estudios previos ${ }^{31}$.

Como era esperable, dexametasona y ACTH no indujeron cambios significativos en las concentraciones de testosterona ${ }^{32}$. La concentración de testosterona tampoco fue afectada por melatonina, apoyando una acción específica de la melatonina sobre la respuesta del cortisol al ACTH.

La disminución de la respuesta de cortisol a ACTH inducida por melatonina no fue debida a un aumento del metabolismo a cortisona, ya que la concentración de cortisona aumentó a valores similares en los sujetos tratados con placebo o con melatonina. Por otra parte, las concentraciones de progesterona, medidas para identificar indirectamente un posible efecto inhibitorio de melatonina sobre la enzima 3ß-hidroxiesteroide dehidrogenasa, aumentaron en respuesta a ACTH, sin demostrarse diferencias entre el grupo placebo y el tratado con melatonina. En cambio, nuestros estudios previos realizados in vitro en corteza suprarrenal de mono capuchino muestran que melatonina inhibe la expresión de la enzima 3ß-hidroxiesteroide dehidrogenasa estimulada por ACTH ${ }^{9,26}$. La diferencia con el presente estudio puede ser 
atribuida a la duración de los protocolos ya que in vitro la exposición a ACTH y a melatonina fue de $48 \mathrm{~h}$. La respuesta de la aldosterona al ACTH fue similar entre el grupo placebo y el tratado con melatonina, lo que sugiere que el efecto inhibitorio de melatonina se ejerce específicamente en la línea glucocorticoidea de la esteroidogénesis.

Varios investigadores han estudiado el efecto de melatonina sobre la respuesta de cortisol inducida por ACTH tanto endógena o como exógena. Perras y cols ${ }^{19}$ no detectaron efectos de melatonina sobre la respuesta de cortisol a la hipoglicemia por insulina al comienzo de la noche. Una situación similar se observó cuando se estimuló con ACTH exógena ${ }^{17-18}$. Considerando la complejidad de la regulación de la corteza de la glándula suprarrenal in vivo ${ }^{20}$ es posible que la presencia de un eje hipotálamo-hipófisis-suprarrenal funcional haya enmascarado los efectos de la melatonina sobre el cortisol plasmático en estos experimentos, lo que explicaría las diferencias con nuestro protocolo, en el que se frenó dicho eje con dexametasona.

Un segundo efecto agudo de la melatonina observado en el presente trabajo fue la prevención de la disminución fisiológica de la prolactina observada en el grupo placebo entre las 08:0010:30 h, sin alcanzar valores de hiperprolactinemia. Una disminución circadiana de la prolactina a estas horas, similar a la observada en el grupo placebo de nuestro estudio, ha sido descrita en voluntarios no tratados con dexametasona ${ }^{33,34}$. Estos resultados concuerdan con los de otros investigadores ${ }^{15,17,19}$ y podrían atribuirse a las

\section{REFERENCIAS}

1. Brzezinski A. Melatonin in humans. N Engl J Med 1997; 336: 186-95.

2. ARENDT J, Skene DJ. Melatonin as a chronobiotic. Sleep Med Rev 2005; 9: 25-39.

3. EkmeKcioglu C. Melatonin receptor in human: biological role and clinical relevance. Biomed Pharmacother 2006; 60: 97-108.

4. Lanoix D, Ouelette R, Vailancourt C. Expression of melatoninergic receptors in human placental choriocarcinoma cell lines. Hum Reprod 2006; 21: 1981-9.

5. Woo MMM, Tai CJ, Kang SK, Nathwani PS, Pang SFP, LEUNG PCK. Direct action of melatonin in human granulosa-luteal cells. J Clin Endocrinol Metab 2001; 86: 4789-97.

6. VAIENTI S, GIUSTI M. Melatonin participates in the control acciones inhibitorias de la melatonina sobre la liberación de dopamina hipotalámica, como ha sido descrita en la rata ${ }^{35}$.

En resumen, este estudio muestra por primera vez la expresión del receptor MT1 de melatonina en la glándula suprarrenal humana y muestra que la ingestión de melatonina disminuye en forma aguda la producción de cortisol estimulada por ACTH en sujetos suprimidos con dexametasona. Aunque el diseño experimental del presente estudio no permite establecer si la reducción de la respuesta de cortisol a ACTH in vivo fue debida a acciones directas sobre la glándula suprarrenal como hemos demostrado anteriormente en otras especies $^{9,10}$, el hecho que esta glándula exprese el receptor MT1 de melatonina abre esta posibilidad.

\section{Agradecimientos}

Agradecemos a: Paola Viviani, magíster en Bioestadística, Centro de Investigaciones Médicas, Facultad de Medicina, Pontificia Universidad Católica de Chile su contribución en los análisis estadísticos de los resultados; Auristela Rojas TM, su asistencia técnica en las determinaciones hormonales por RIA; Dra. Doris Haack, Departamento de Farmacología, Universidad de Heidelberg, Alemania por proporcionarnos los anticuerpos para medir cortisol y cortisona.

Este trabajo fue financiado por el proyecto VRAID N5/2006 de la Pontificia Universidad Católica de Chile y fondos del Departamento de Endocrinología, Facultad de Medicina, Pontificia Universidad Católica de Chile.

of testosterone secretion from rat testis: an overview of our experience. Ann NY Acad Sci 2002; 966: 284-9.

7. Frungieri MB, Mayerhofer A, Zitta K, Pignataro OP, Calandra RS, Gonzálfz-Calvar SI. Direct effect of melatonin on Syrian hamster testes: Melatonin subtype 1a receptors, inhibition of androgen production, and interaction with the local corticotropin-realising hormone system. Endocrinology 2005; 146: 1541-52.

8. Richter HG, Torres-Farfán C, García-Sesnich J, AbarzÚa-Catalán L, Henríquez MG, Alvarez-Felmer M et al. Rhythmic expression of functional MT1 melatonin receptors in the rat adrenal gland. Endocrinology 2008; 149: 995-1003.

9. Torres-Farfán C, Richter HG, Germain A, Valenzueia G, Campino C, Rojas-García P ET aL. Matemal melatonin selectively inhibits cortisol production in the primate fetal adrenal gland. J Physiol 2004; 554: 841-56. 
10. Torres-Farfán C, Richter HG, Rojas-García P, Vergara M, FORCELEDO ML, VALADARES LE ET AL. mtl Melatonin receptor in the primate adrenal gland: inhibition of adrenocorticotropin-stimulated cortisol production by melatonin. J Clin Endocrinol and Metab 2003; 88: 450-8.

11. ValenZueita FJ, Torres-Farfán C, Richter HG, MÉndez N, CAmpino C, Torrealba F ET AL. Clock gene expression in adult primate suprachiasmatic nuclei and adrenal: is the adrenal a peripheral clock responsive to melatonin? Endocrinology 2008; 149: 1454-61.

12. Weibel $L$, BRANDENBERGER $G$. The start of the quiescent period of cortisol remains phase locked to the melatonin onset despite circadian phase alterations in humans working the night schedule. Neurosci Lett 2002; 318: 89-92.

13. Leproult $R$, Colecchia EF, L'Hermite-Baleriaux M, Van CAUTER E. Transition from dim to bright light in the morning induces an immediate elevation of cortisol levels. J Clin Endocrinol Metab 2001; 86: 151-7.

14. SCHEER FAJL, Bujs RM. Light affects moming salivary cortisol in humans. J Clin Endocrinol Metab 1999; 84: 3395-8.

15. WALDhAuser F, LuEberman HR, LyNCh HJ, WaLdHAUSER M, HERKNER K, FRISCH H ET AL. A pharmacological dose of melatonin increases PRL levels in males without altering those of $\mathrm{GH}, \mathrm{LH}, \mathrm{FSH}, \mathrm{TSH}$, testosterone or cortisol. Neuroendocrinology 1987; 46: 125-30.

16. Cagnacci A, Soldani R, Yen SSC. Melatonin enhances cortisol levels in aged but not young women. Eur J Endocrinol 1995; 133: 691-5.

17. Terzolo M, Piovesan A, Osella G, Torta M, Bunita T, Paccotti P ET al. Exogenous melatonin enhances the TRH-induced prolactin release in normally cycling women: a sex-specific effect. Gynecol Endocrinol 1991; 5: 83-94.

18. Paccotti P, Terzolo M, Piovesan M, Torta M, Vignani A, ANGeL A. Effects of endogenous melatonin on human pituitary and adrenal secretions. Hormonal responses to specific stimuli after acute administration of different doses at two opposite circadian stages in men. Chronobiologia 1988; 15: 279-88.

19. Perras B, Özcan S, FeHm HL, Born J. Melatonin does not inhibit hypothalamic-pituitary-adrenal activity in waking young men. J Neuroendocrinol 2005; 17: 811-6.

20. BorNSTEIN SR, Chrousos GP. Adrenocorticotropin (ACTH) and Non-ACTH-mediated regulation of the adrenal cortex: Neural and Immune Inputs. J Clin Endocrinol Metab 1999; 84: 1729-36.

21. Drew JE, Wimams LM, Hannah LT, Barrett P, AbramoVICH DR. Melatonin receptors in the human fetal kidney: 2-[125I]iodomelatonin binding sites correlated with expression of Mella and Mel1b receptor genes. J Endocrinol 1998; 156: 261-7.

22. PARK YJ, PARK KS, Kim JH, ShIN CS, Kim SY, LeE HK. Reproducibility of the cortisol response to stimulation with the low dose (1microg) of ACTH. Clin
Endocrinol 1999; 51: 153-8.

23. Torres-Farfán C, ValenZueita FJ, EBensperger R, MÉndez N, CAMPINo C, RichteR HG ET AL. Circadian cortisol secretion and circadian adrenal responses to ACTH are maintained in dexamethasone suppressed capuchin monkeys (Cebus apella). Am J Primatol 2008; 70: 93-100.

24. Engeland WC, ARnhold MM. Neural circuitry in the regulation of adrenal corticosterone rhythmicity. Endocrine 2005; 28: 325-32.

25. Torres-Farfán C, Rocco V, Monsó C, Valenzueia FJ, Campino C, Germain A et AL. Maternal melatonin effects on clock gene expression in a nonhuman primate fetus. Endocrinology 2006; 147: 4618-26.

26. Torres-Farfán C, Valenzuela FJ, Germain AM, Viale ML, Campino C, Torrealba F ET al. Maternal melatonin stimulates growth and prevents maturation of the capuchin monkey fetal adrenal gland. J Pineal Res 2006; 41: 58-66.

27. Sufi SB, Donaldson A, JefFcoate SL. Programme for the provision of matched assay reagents for the radioimmunoassay of hormones in reproductive physiology. Methods manual 1993. London: WHO.

28. LtTell RC, MiuKen GA, Stroup WW, Wolfinger RD, SCHABENBERGER O. Analysis of repeated measures data. In SAS for mixed models. $2^{\text {nd }}$ ed, Ed. SAS Institute 2006; 159-204.

29. Reppert SM, Weaver DR, Ebisawa T. Cloning and characterization of a mammalian melatonin receptor that mediates reproductive and circadian responses. Neuron 1994; 13: 1177-85.

30. Fischer S, Smolnik R, Herms M, Born J, Fehm H. Melatonin acutely improves the neuroendocrine architecture of sleep in blind individuals. J Clin Endocrinol Metab 2003; 88: 5315-20.

31. Fardella CE, Mosso L, Gómez-Sánchez C, Cortés P, Soto J, Gómez L et aL. Primary hyperaldosteronism in essential hypertensives: prevalence, biochemical profile, and molecular biology. J Clin Endocrinol Metab 2000; 85: 1863-7.

32. Campusano C, Brusco F, Campino C, Rodríguez L, ARTEaga E. Comparación de distintos métodos para evaluar la función androgénica en el adulto mayor. Rev Méd Chile 2006; 134: 1123-8.

33. Van Cauter E, L'Hermite M, Copinschi G, Refetoff S, Desir D, Robyn C. Quantitative analysis of spontaneous variations of plasma prolactin in normal man. Am J Physiol 1981; 241: E355-E363.

34. Waldstreicher J, Duffy JF, Brown EN, Rogacz S, Aluan JS, CzeISLER CA. Gender differences in the temporal organization of prolactin (PRL) secretion: evidence for a sleep-independent circadian rhythm of circulating PRL levels- a clinical research center study. J Clin Endocrinol Metab 1996; 81: 1483-7.

35. ZISAPEL N. Melatonin-dopamine interactions: from basic neurochemistry to a clinical setting. Cell Mol Neurobiol 2001; 21: 605-16. 